\title{
Nonlinear Technique for the Analysis of the Free-Running Oscillator Phase Noise in Presence of an Interference Signal
}

\author{
Sergio Sancho, Mabel Ponton, Almudena Suarez \\ University of Cantabria, Santander, Spain.
}

\begin{abstract}
A new methodology for the prediction of oscillator phase noise under the effect of an interference signal is presented. It is based on a semi-analytical formulation in the presence of the noisy interferer, using a realistic oscillator model extracted from harmonic-balance simulations. The theoretical analysis of the phase process enables the derivation of key mathematical properties, used for an efficient calculation of the interfered-oscillator phase noise spectrum. The resulting quasiperiodic spectrum is predicted, as well as the impact of the interferer phase noise over each spectral component, in particular over the pulled oscillation frequency. It is demonstrated that under some conditions, the phase noise at this component is pulled to that of the interference signal. Resonance effects at multiples of the beat frequency are also predicted. The analyses have been validated with experimental measurements, obtaining excellent agreement.
\end{abstract}

Index Terms - Microwave oscillator, frequency-domain analysis, interferer, injection pulling, phase noise.

\section{INTRODUCTION}

A well-known problem in communication systems is the pulling of the local-oscillator frequency by an interferer. In phase-locked loops (PLLs), this unwanted frequency shift can unlock the voltage-controlled oscillator (VCO) from its low frequency reference [1]. Synchronization to the interference may also occur, which in the PLL would lead to a mixing of the reference frequency and the synchronized-oscillation frequency. Though these effects have been studied in previous work, little effort has been devoted to investigate the impact of the interferer signal and its power spectral density on the interfered oscillator phase noise. This characteristic is of critical importance in communication systems, as it can give rise to demodulation errors. Predicting its degradation in the presence of an interferer will help correct the prototypes at the design stage, so as to make them more robust against the interferer action.

Here an analysis of the effect of an interferer on the phasenoise spectrum of an oscillator circuit is presented. To have an impact on this spectrum, the interferer frequency must be close to that of the interfered oscillator, as otherwise the two signals will have independent phase variations. The analysis is involved since in the general case of an oscillator that is not locked to the interferer, the solution will be quasi-periodic, with two fundamental frequencies: the oscillation frequency, affected by the interferer, and the interferer frequency. Ordinary phase-noise analysis methods of oscillator circuits are unable to consider an additional fundamental. The only way to address the problem would be through a two tone analysis, plus phase-noise analysis of the oscillator carrier, which has failed to converge in commercial harmonic balance
(HB). Instead, a semi-analytical method is applied here. A realistic model of the interfered oscillator is used, based on derivatives obtained through finite differences in $\mathrm{HB}$, calculated about the free-running point [2-4]. The analysis will be based on the determination of the phase perturbation of the interfered oscillator in the presence of the undesired signal spectrum, as well as its own noise sources.

\section{ANALYSIS OF THE QUASI-PERIODIC STATE}

The analysis will be illustrated through its application to the FET-based oscillator at $2.5 \mathrm{GHz}$ in Fig. 1. The interference signal is modeled as a current source, in parallel with the oscillator output resistor of $50 \Omega$. Interference signal entering through the output port is a realistic situation in front-end systems. However, the formulation can be equally applied for other locations of the interference equivalent source. It will be derived in terms of the voltage signal $v(t)$ at the oscillator drain terminal. Note that the analysis can be performed at a node different from the one where the interferer current source is connected, since its effect is modeled by means of transfer functions [2-4].

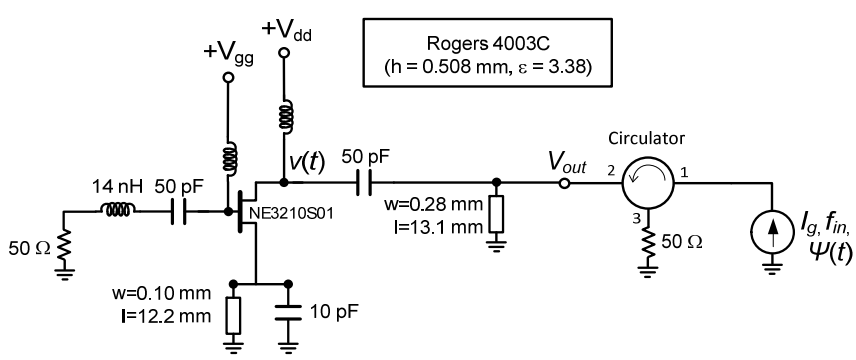

Fig. 1 Schematic of the interfered oscillator operating at $2.5 \mathrm{GHz}$ based on the FET NE3210S01. The interference is modeled with a current source and introduced into the oscillator through a circulator

In this preliminary work, the interferer will be assumed to be a small amplitude single-tone current source with its own phase-noise process $\psi(t)$. This signal is expressed as $i_{g}(t)=2 \operatorname{Re}\left\{I_{g} e^{j\left[\omega_{i n} t+\psi(t)\right]}\right\}$ with $\omega_{i n}=2 \pi f_{\text {in }}$. The introduction of the source $i_{g}(t)$ perturbs each $k$-th harmonic component of the signal $v(t)$ :

$$
v(t)=\sum_{k} v_{k}(t), v_{k}(t)=\left[V_{k}+\Delta V_{k}(t)\right] e^{j \phi_{k}(t)} e^{j k \omega_{i n} t}=X_{k}(t) e^{j k \omega_{i n} t}
$$

where each $v_{k}(t)$ is a narrow band process centered at the frequency component $k f_{i n}$. While the harmonic amplitudes $V_{k}$ 
are slightly perturbed by the interferer, the phase components $\phi_{k}(t)$ are unbounded in the non-synchronized state [1]. Now, following the semi-analytical formulation technique (SAF) proposed in [2-4], the Implicit Function Theorem is applied to obtain an envelope-domain model of the oscillator dynamics in the form of a single outer-tier equation:

$$
Y\left[V_{1}+\Delta V_{1}, j\left(\omega_{o}+\Delta \omega\right)+s, I_{g} \cos \psi(t), I_{g} \sin \psi(t)\right] e^{j \phi(t)}=\frac{I_{n}(t)}{V_{1}+\Delta V_{1}}
$$

where $Y$ is the first harmonic total admittance at the observation node, $V_{1}$ is the first harmonic amplitude and $\Delta \omega=2 \pi \Delta f=\omega_{o}-\omega_{i n}$, where $\omega_{o}=2 \pi f_{o}$ is the free-running frequency. The phase $\phi(t) \equiv \phi_{1}(t)$ represents the first-harmonic phase shift from the interferer frequency $f_{\text {in }}$. Using the technique in [5], the effect of all the noise sources existing in the circuit has been modeled with an equivalent current generator $i_{n}(t)=\operatorname{Re}\left\{I_{n}(t) e^{j \omega_{i n} t}\right\}$ connected in parallel at the transistor drain. In the absence of noise sources, if the freerunning oscillation at $f_{o}$ does not synchronize to the interferer, system (2) provides a quasi-periodic solution with one autonomous component at $f_{o}$ [6] Note that in the case of a phase-locked loop (PLL) or an injection-locked oscillator the situation would be different. In those cases, the admittance function $Y$ has a dependence on the amplitude and phase components of the reference or external generator signal [5, 7]. This dependence removes the system autonomy of the interfered system quasi-periodic solution [8]. Therefore, the interfered system does not remain invariant under phase shifts, providing different phase noise results than the autonomous case, which will be the one analyzed here.

Assuming small interferer amplitude, equation (2) can be approximated by a first-order Taylor series about the freerunning state, which yields the following equation for the phase shift [4]:

$$
\begin{aligned}
& \dot{\phi}=\Delta \omega+K_{s} \sin (\phi-\psi)+K_{c} \cos (\phi-\psi)+\varepsilon(t), \\
& \varepsilon(t)=H_{r} I_{n}^{r}+H_{i} I_{n}^{i}
\end{aligned}
$$

In [4], the coefficients $\left(K_{c}, K_{s}, H_{r}, H_{i}\right)$ are provided in terms of $I_{g}$ and the derivatives of the admittance function (2) evaluated at the free-running solution, together with a technique to calculate these derivatives through finite differences in commercial HB. The coefficients $K_{c}, K_{s}$ contain, among others, the admittance derivatives with respect to the real and imaginary parts of the current source phasor that models the interferer, acting as transfer functions. Equation (3) provided by the SAF technique can be used to calculate the oscillator phase noise in the presence of a noisy interferer. The study must be preceded by the analysis of the steady-state solution, in the absence of noise sources.

\section{A. Phase dynamics in the absence of noise sources}

In the absence of noise sources, Equation (3) represents an autonomous system that can be written as:

$$
\dot{\phi}=\Delta \omega+K_{s} \sin \phi+K_{c} \cos \phi \equiv g(\phi)
$$

The phase shift becomes a constant value $\phi=\phi_{s}$, fulfilling $g\left(\phi_{s}\right)=0$, only when the free-running frequency synchronizes to the interferer. Then, in the more general unsynchronized conditions $g(\phi) \neq 0, \forall \phi$ must be fulfilled, implying that $\phi(t)$ grows or decreases monotonically. Let us assume that $g(\phi)>0, \forall \phi$. The case $g(\phi)<0, \forall \phi$ is symmetric. The time required by the phase variable to pass through an arbitrary interval $\left[\phi_{\mathrm{a}}, \phi_{b}\right]$ is given by:

$$
\begin{aligned}
& T\left(\phi_{a}, \phi_{b}\right)=\int_{\phi_{a}}^{\phi_{b}} \frac{d \phi}{g(\phi)}=u_{0}\left(\phi_{b}-\phi_{a}\right)+q\left(\phi_{b}\right)-q\left(\phi_{a}\right), \\
& u(\phi) \equiv \frac{1}{g(\phi)}=u_{0}+\tilde{u}(\phi), \quad u_{0}=\frac{1}{2 \pi} \int_{0}^{2 \pi} u(\phi) d \phi, \\
& q(\phi) \equiv \int \tilde{u}(\phi) d \phi, \quad q(\phi)=q(\phi \pm 2 \pi)
\end{aligned}
$$

From the definition of $g(\phi)$, the phase shift fulfills:

$T_{b} \equiv T(\phi, \phi+2 \pi)=u_{0} 2 \pi+q(\phi+2 \pi)-q(\phi)=u_{0} 2 \pi, \forall \phi$

Property (6) implies that $\dot{\phi}(t)$ is periodic, since:

$\dot{\phi}\left(t+T_{b}\right)=g\left[\phi\left(t+T_{b}\right)\right]=g[\phi(t)+2 \pi]=g[\phi(t)]=\dot{\phi}(t)$

Considering (6)- (7), the phase variable can be expressed as:

$$
\phi(t)=\omega_{b} t+\sum_{k=-N}^{N} P_{k} e^{j k \omega_{b} t}, \quad \omega_{b}=2 \pi f_{b}=2 \pi / T_{b}
$$

Now, introducing expression (8) in the process $v_{1}(t)$ in (1) one obtains:

$$
\begin{aligned}
v_{1}(t) & =X_{1}(t) e^{j \omega_{i n} t} \approx V_{1} e^{j \phi(t)} e^{j \omega_{i n} t} \\
& =V_{1} e^{j\left(\omega_{i n}+\omega_{b}\right) t} \exp \left\{\sum_{k=-N}^{N} P_{k} e^{j k \omega_{b} t}\right\} \\
& =\sum_{k} X_{1}^{k} e^{j\left(\omega_{i n}+k \omega_{b}\right) t}
\end{aligned}
$$

Equation (9) implies that, for a small amplitude interferer, $v_{1}(t)$ is a multi-tone signal containing the intermodulation products $f_{k}=f_{\text {in }}+k f_{b}$. The oscillator frequency is pulled from $f_{o}$ to $f_{o}^{\prime} \equiv f_{1}=f_{\text {in }}+f_{b}$. This theoretical result has been verified by simulating the spectrum of the time-varying first harmonic component $X_{1}(t) \approx V_{1} e^{j \phi(t)}$ using the envelopetransient method in commercial HB software. This simulation is compared in Fig. 2 with the result of equation (9). Here, the components $\left\{f_{b}, P_{0}, \ldots P_{N}\right\}$ have been calculated in the frequency domain by introducing expression (8) in equation (4) and solving the resulting HB system. 


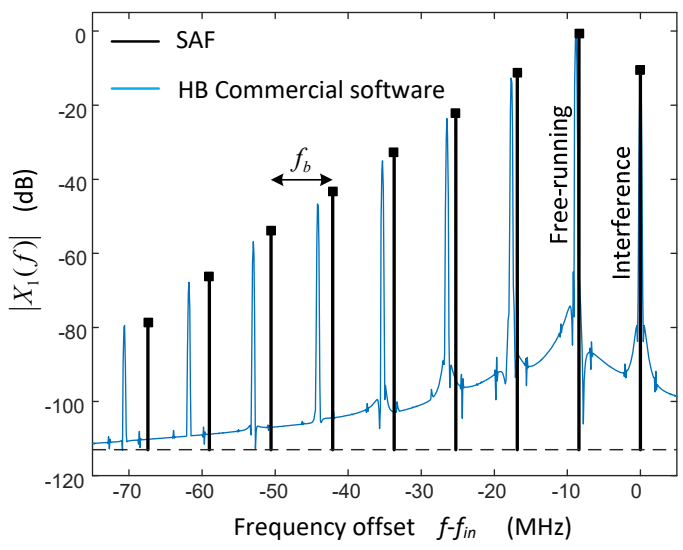

Fig. 2. Spectrum of the first harmonic component $X_{1}(t) \approx V_{1} e^{j \phi(t)}$ in the presence of an interferer with $I_{g}=0.2 \mathrm{~mA}$ and $\Delta f=-10 \mathrm{MHz}$.

\section{B. Phase noise analysis}

Let $\phi(t)=\phi 0(t)$ be the phase shift in absence of noise sources. The noisy components introduced by the local sources and the interferer perturb the steady state $\phi_{0}(t)$ in the form $\phi(t)=\phi_{0}(t)+\Delta \phi(t)$. Assuming that $\Delta \phi(t)$ is small, equation

(3) can be linearized about $\phi_{0}(t)$ obtaining:

$$
\Delta \dot{\phi}(t)=b_{0}(t)[\Delta \phi(t)-\psi(t)]+\varepsilon(t), \quad b_{0}(t) \equiv \frac{d g\left[\phi_{0}(t)\right]}{d \phi}
$$

Equation (10) shows that the phase perturbation is a cyclostationary process governed by the periodic function $b_{0}(t)$. Then, the phase perturbation can be expressed as:

$$
\Delta \phi(t)=\sum_{k} \Phi_{k}(t) e^{j k \omega_{b} t}
$$

where each time-varying harmonic $\Phi_{k}(t)$ is a narrow-band stochastic process. Equations (9) and (11) show that the phase noise about each component at $f_{k}$ is the result of the contributions of all the components $\Phi_{k}$. In particular, the main contribution to phase noise about the pulled frequency $f_{o}{ }^{\prime} \equiv f_{1}$ is given by $\Phi_{0}$. This is because the contributions of the components $\Phi_{k}$ for $k \neq 1$ are scaled by the factors $\left|X_{1}^{k} / X_{1}^{1}\right|<<1$. Then, the phase noise about $f_{o}{ }^{\prime}$ can be approximated by the power spectral density of the process $\Phi_{0}(t)$, assumed to be stationary. The conversion between the interferer phase noise $\psi(t)$ and $\Phi_{0}(t)$ is determined by the harmonic components $\left\{B_{k}\right\}$ of $b_{0}(t)$. In particular, the dc component of this signal is:

$$
\begin{aligned}
B_{0} & =\frac{1}{T_{b}} \int_{0}^{T_{b}} b_{0}(t) d t=\frac{1}{T_{b}} \int_{0}^{T_{b}} \frac{d g\left[\phi_{0}(t)\right]}{d \phi} d t=\frac{1}{T_{b}} \int_{g(0)}^{g\left(T_{b}\right)} \frac{d g}{g}= \\
& =\frac{1}{T_{b}} \log \frac{g\left(T_{b}\right)}{g(0)}=\frac{1}{T_{b}} \log \frac{\dot{\phi}_{0}\left(T_{b}\right)}{\dot{\phi}_{0}(0)}=0
\end{aligned}
$$

where $g=d \phi_{0} / d t$ has been applied. As a consequence, there is no direct conversion from the interferer phase noise $\psi(t)$ to $\Phi_{0}(t)$. The rest of the coefficients $B_{i}$ determine the noise conversion between all the harmonic components $f_{k}$, represented in Fig. 2, as gathered from (10). In this preliminary work, the analysis has been limited to the coefficient $B_{1}$, translating equation (10) to the frequency domain to approach the phase noise about $f_{o}$ ' by:

$$
\left\langle\left|\Phi_{0}(f)\right|^{2}\right\rangle \approx\left(\frac{\alpha}{\alpha+4 \pi^{2}\left(f^{2}-f_{b}^{2}\right)}\right)^{2}\left\langle|\psi(f)|^{2}\right\rangle+\frac{\left\langle|\varepsilon(f)|^{2}\right\rangle}{4 \pi^{2} f^{2}}
$$

where $\alpha=2\left|B_{1}\right|^{2}$ acts as a sensitivity coefficient that determines the influence of the interferer phase noise, $f$ is the frequency offset from the carrier at $f_{o}$ ' and the second term is the oscillator's own noise. In the absence of an interferer $\alpha$ becomes zero and equation (13) agrees with the free-running oscillator phase noise. The introduction of the interferer provides $\alpha>0$, increasing the level of the phase noise characteristic, since it adds a term proportional to the interferer phase noise. The interferer influence is most noticeable when its phase noise characteristic is higher than that of the free-running oscillator. In that case, equation (13) predicts that the phase noise characteristic about $f_{o}$ ' is pulled towards the interferer phase noise curve. This behavior has been verified in Fig. 3, where the measured phase noise spectrum and the one predicted by equation (13) (SAF) have been compared. The interferer is not represented. Instead, its measured phase noise from zero offset frequency is traced for comparison. As predicted by (13), the oscillator phase noise is pulled to that of the interferer. Equation (13) also demonstrates a resonance effect, with maximum phase noise at an offset frequency about $f_{b}$. Note that the singularity predicted by (13) at $f_{b}$ is fully consistent with the presence of a steady-state spectral line at $f_{2}=f_{o}^{\prime}+f_{b}$. The noise resonances at frequency offsets $k f_{b}$ for $k>1$ could be obtained by considering the corresponding coefficients $B_{k}$ when translating equation (10) to the frequency domain. Measurements are superimposed with a very good agreement.

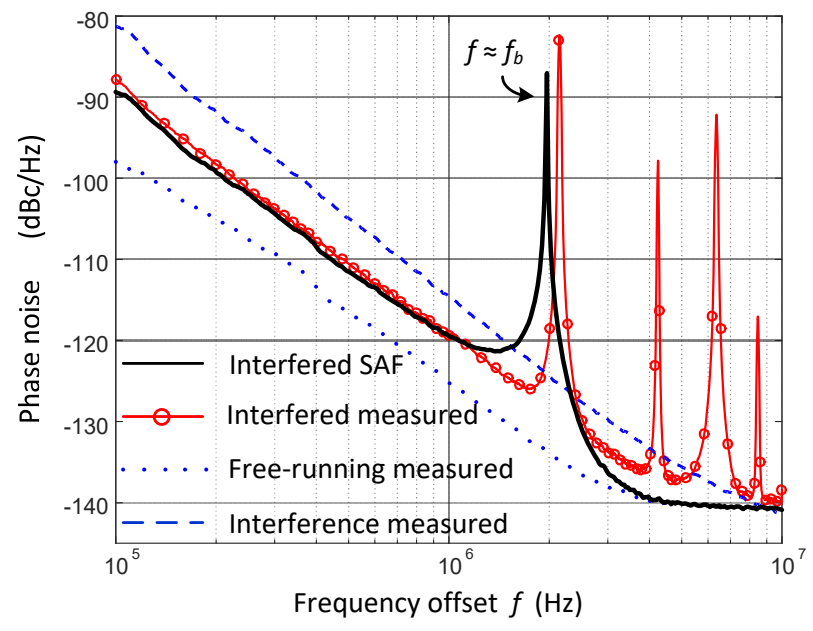

Fig. 3. Phase noise in the presence of an interferer with $P_{\text {in }}=-29.6 \mathrm{dBm}$ and $\Delta f \approx-2 \mathrm{MHz}$. The phase noise characteristic about $f_{o}$ ' is pulled towards the interferer phase noise. 


\section{CONCLUSION}

The influence of the phase noise of an interferer on freerunning oscillator phase noise has been studied. A semianalytical formulation has been derived, which leads to a simple equation that predicts the pulling effect of the interfered oscillator phase noise towards that of the interference signal. This prediction has been verified by measurements in a FET-based oscillator at $2.5 \mathrm{GHz}$ in the presence of an interferer at $2 \mathrm{MHz}$ offset.

\section{ACKNOWLEDGEMENT}

This work was supported by the Spanish Ministry of Economy and Competitiveness under the research project TEC2014-60283-C3-1-R, the European Regional Development Fund (ERDF/FEDER) and Juan de la Cierva Research Program IJCI-2014-19141, and by the Parliament of Cantabria under the project Cantabria Explora 12.JP02.64069.

\section{REFERENCES}

[1] B. Razavi, "A study of injection locking and pulling in oscillators," IEEE J Solid State Circuits, vol. 39, pp. 1415-1424, 2004.

[2] A. Suarez, Analysis and Design of Autonomous Microwave Circuits. Wiley, 2009,

[3] J. Dominguez, A. Suarez and S. Sancho. "Semi-analytical formulation for the analysis and reduction of injection-pulling in front-end oscillators," Microwave Symposium Digest, 2009. MTT '09. IEEE MTT-S International, 2009, pp. 1589-1592.

[4] S. Sancho, F. Ramírez and A. Suárez, "Stochastic Analysis of Cycle Slips in Injection-Locked Oscillators and Analog Frequency Dividers," IEEE Transactions on Microwave Theory and Techniques, vol. 62, pp. 3318-3332, 2014.

[5] F. Ramirez, M. Ponton, S. Sancho and A. Suarez, "Phase noise of injection-locked oscillators and frequency dividers," IEEE Transactions on Microwave Theory and Techniques, vol. 56, pp. 393-407, Feb. 2008.

[6] E. De Cos, A. Suarez and S. Sancho, "Envelope transient analysis of self-oscillating mixers," IEEE Transactions on Microwave Theory and Techniques, vol. 52, pp. 1090-1100, 2004.

[7] M. Ponton, E. Fernandez, A. Suarez and F. Ramirez, "Optimized Design of Pulsed Waveform Oscillators and Frequency Dividers," IEEE Transactions on Microwave Theory and Techniques, vol. 59, pp. 3428-3440, 2011.

[8] A. Suarez and R. Melville, "Simulation-assisted design and analysis of varactor-based frequency multipliers and dividers," IEEE Transactions on Microwave Theory and Techniques, vol. 54, pp. 1166-1179, 2006. 